\title{
Experimental Study in Kinematics of Rogue Wave
}

\author{
Hae-jin Choi*, Kwang Hyo Jung**, Sungbu Suh**, Seung Jae Lee***, Hyo Jae Jo***, and Han Suk Choi**** \\ *Samsung Engineering Corporate Limited, Seoul, Korea, \\ **Development of Naval Architecture and Ocean Engineering, Dong-Eui University, Busan, Korea \\ ***Division of Naval Architecture and Ocean Systems Engineering, Korea Maritime University, Busan, Korea \\ ****Department of Naval Architecture and Ocean Engineering, Pusan National University, Busan, Korea
}

KEY WORDS: Rogue wave, Velocity, Local and convective accelerations, Wave crest, Wave theory

ABSTRACT: The rogue wave was generated in a two-dimensional wave tank the rogue wave kinematics was investigated including local and convective accelerations of the water particle and verification of existing prediction methods. PIV technique was applied to measure the wave kinematics near the wave crest which extended to compute the local and convective accelerations. The experimental results were compared with several analytical predictions. The convective acceleration under the crest of rogue wave has a similar magnitude with the local acceleration.

\section{Introduction}

Since George Biddell Airy's long and influential article "Tides and Waves", was published in 1845, many research has been done to look for the simulation of ocean waves. The understanding of water waves and the associated kinematics has advanced substantially during the last four to five decades. There have also been a number of experimental studies in which wave characteristics have been measured within a laboratory wave flume. Experimental study in a laboratory provides substantial advantages in a water wave study, such as the control of wave parameters, the repeatability, and the relatively low cost. The nonlinearity and the directionality of water waves are crucial elements to an adequate understanding of water force, especially for the high waves generated by complex wind fields such as tropical storms. However, the phenomena of ocean waves are hard to be explained with theoretical predictions based on a regular wave, a uni-directional irregular wave, or a multidirectional short crest wave.

Within the past 20 years, at least 200 supertankers longer than $200 \mathrm{~m}$ long have been lost. The causes of many cases are believed to 'rogue waves' which are waves of exceptional height and abnormal shape. There are several reports about suddend is asters in extreme waves. For example, two large Norwegian bulkships M/S "NorseVariant" and M/S"Anita" disappeared at the same time and location. According to the conclusion of the Court of Inquiry, a very large wave suddenly broke several hatch covers on deck, and the ships were filled with water and sank before any emergency call was given. The wave that caused loss of both ships was probably a freak or rogue wave (Kjeldsen, 2001). Cruise ship damage had been rare, but recently some cruise liners have been hit hard by rogue waves. The Queen Elizabeth II, for example, was struck by a $29 \mathrm{~m}$ rogue wave in 1995 in the North Atlantic (Met Office, 1996). The Caledonian Star, sailing in the South Atlantic in 2001, was hit by a rogue wave estimated at $30 \mathrm{~m}$. The Explorer, on a "semester-at-sea" sailing in the North Pacific, was damaged in January 2005 when the ship, carrying nearly 1000 people including almost 700 college students, was struck by a wave estimated to be $17 \mathrm{~m}$ in height. The wall of water smashed into the bridge of the $180 \mathrm{~m}$ long ship. These well-built cruise ships suffered little damage and had few injuries from the attack of rogue waves (Mastroianni, 2005). Most recently, the Norwegian Dawn, a 3-year-old $294 \mathrm{~m}$ long cruise ship carrying more than 2200 passengers and heading back to New York from the Bahamas, was pounded by a rogue wave during a storm in April 2005 off the South Carolina coast. The wave reached the $10^{\text {th }}$ deck of the towering ship and shattered two windows. As a result, 62 cabins were flooded and some public areas were damaged, but only four people were injured, according to the cruise line (Lemire,2005). The rogue wave was estimated to be $21 \mathrm{~m}$, far higher than surrounding waves. Offshore platforms have also been struck. On January 1, 1995, the Draupner oil rig in the North Sea was hit by a whose wave whose height was $26 \mathrm{~m}$ measured by an onboard laser wave-meter. with the surrounding waves reaching $12 \mathrm{~m}$ (Clauss, 2002).

Many marine scientists have clung to statistical models that explain rogue waves as a monstrous deviation which could occur only once in a thousand years. Had the ships

Corresponding author Kwang Hyo Jung: Busan Busanjingu Eonkwangro 995, 051-890-2592, Khjung@deu.ac.kr 
encountered the 1000-year storm? So how many 1000-year storms have there been and how common are they? MaxWave, a German scientific group, examined 30,000 worldwide satellite photos taken by the European Space Agency (ESA). According to MaxWave, 10 rogue waves, each more than $25 \mathrm{~m}$ in height, were identified around the globe within the short three-week research period in 2001 (Rosenthal and Lehner, 2004). These rogue waves, far bigger than any surrounding waves, can occur during storms or calmer seas, and almost anywhere, but it appears they occur more frequently where there are strong currents, such as the Gulf Stream off the eastern coast of North America.

Rogue waves, different from tsunamis that result from earthquakes, are formed in three ways. They grow from strong winds beating against an opposing ocean current, from intersecting waves driven together by storms or from swells having their energy focused by the topography of the sea floor. Whether they are wind-driven waves, currents, ocean bottom topography or inclement weather, these factors can play a role in rogue wave development. Many researchers (Bonmarin and Kjeldsen, 2001; Olagnon and Iseghem, 2001) defined rogue waves as waves whose height is twice the 'significant wave height' of surrounding waves.

During the last decade, extreme waves have drawn considerable attention from media and scientific and ocean engineering communities. There have been episodes of severe damage to offshore structures and ships with few observations of surprisingly large individual waves. Notably, Draper (1965) suggested using the term "freak waves" and developed a theory for application to a real ocean wave spectrum. Although a large number of studies related to freak waves were carried out, no authoritative definition of "freak waves", which represented the observed abnormal waves such as the New Year Draupner wave. Even the terms for such waves varied as extreme waves, giant waves, mountain waves, exceptional high waves, rogue waves, etc. Recently many researchers have used "rogue waves" as academic terms for freak waves. The use of the term "rogue waves" will also be applied in this study. Many researchers (Dean, 1990; Faukner, 2001; Kjeldsen, 2001; Olagnon and Iseghem, 2001; Haver, 2001) have commonly used the criteria to define rogue waves: waves exceeding a height of $2 \mathrm{Hs}$, where $\mathrm{Hs}$ is the significant wave height.

The causes of rogue waves in the real ocean have long been investigated and many hypothetical mechanisms have been proposed from different points of view and corresponding techniques. Over the last decade, great progress has been made in the understanding of physical mechanisms of rogue wave phenomenon. With the assumption of the linear wave theory, rogue waves can be considered as the sum of a very large number of independent monochromatic waves with different frequencies and directions. A rogue wave may appear in the process of geometrical focusing, dispersion enhancement (Kharif et al., 2001; Smith and Swan, 2002) and wave-current interactions (Peregrine, 1976; Smith, 1976; Lavrenov, 1998). The combination of the geometrical focusing and dispersion enhancement mechanism to form an extreme wave has also been examined by $\mathrm{Wu}$ and Nepf (2002).

Nonlinear theory also suggested the mechanisms of rogue wave formation. All three processes mentioned above are investigated analytically and numerically in the framework of weakly nonlinear models like the nonlinear Schrödinger equation (Trulsen and Dysthe, 1997; Henderson et al., 1999; Dysthe and Trulsen, 1999; Osborne et al., 2000; Onorato et al., 2002), as well as in the laboratory (Tulin and Waseda, 1999). Nonlinear wave-wave interaction has been addressed in association with rogue wave formation (Mori and Yasuda, 2002; Jansen, 2003).

The phenomenon of rogue waves has been studied using a higher-order nonlinear and dispersive model such as the fully nonlinear potential equations. Grue (2002), Clamond and Grue (2002) performed a fully nonlinear numerical simulation of the long time evolution of a two-dimensional localized long wave packet. A few studies have been conducted for rogue waves in two main areas: generating rogue wave in laboratory conditions and defining the criteria of rogue waves. Wu and Yao (2004) investigated rogue wave kinematics by generating a rogue wave in the two-dimensional wave tank using a combined mechanism of dispersion enhancement and wave-current interaction. Kim and Kim (2003) simulated a Draupner rogue wave in the two-dimensional wave tank and measured the horizontal particle velocity using LDV and horizontal force on a vertical truncated cylinder fixed in the wave. Zou and Kim (2000) generated a strongly asymmetric wave in the two-dimensional wave tank. They adopted two steps which were time distortion and crest distortion to the highest wave in irregular wave train. The definition of rogue wave, Hmax/Hs>2.0, is challenged due to its short comings in representing the full spectrum of the surrounding sea states. In this study, the rogue wave was generated in a two-dimensional wave tank and investigated the rogue wave kinematics including local and convective accelerations of water particle. The water particle velocity components of regular waves were measured and compared to verify the methodology using the PIV technique. 


\section{Experimental Set-up Condition}

To measure the water particle velocities of water waves, a series of experiments was conducted in 2-D wave tank which is $35 \mathrm{~m}$ long, $0.91 \mathrm{~m}$ wide, and $1.22 \mathrm{~m}$ deep glass-walled flume as shown in Fig. 1. It was equipped with a permeable wave absorbing 1:5.5 sloped beach and its reflection coefficient was $5 \%$. Wave maker had a dry-back, hinged flap type and was driven by a synchronous servo-motor controlled by a computer and hydro-statically balanced using an automatic near constant force and a pneumatic control system. The PIV technique was employed to obtain the velocity field of water waves. The PIV system and the wavemaker were synchronized by computer A housing a data acquisition board (National Instruments AT-AO-6/10) which generated analog output DC voltage (Fig. 1). The timing of laser pulses was controlled by computer $\mathrm{C}$ housing the Programmable-Timing-Unit-Board. Also, the PIV system and the wave maker were synchronized with the wave elevation data from the wave gages.

The digital CCD camera mounted with a $105 \mathrm{~mm} f / 1.8$ micro focal lens set at $f / 2.8 \sim 4.0$ was used to obtain PIV images. It had $1280 \times 1024$ pixels, $6.7 \times 6.7 \mathrm{~mm} /$ pixel size, 12 bit dynamic range, and $8 \mathrm{~Hz}$ framing rate. The PIV images were recorded by the double-frame/single-pulsed method. The time difference $(d t)$ between the 1 stframe and $2^{\text {nd }}$ frame was adjusted to be about 3 5ms, which was determined by the maximum displacement to be less than a third of the width of the interrogation window size. A pair of images (1280x1024pixels, $d t=3 \mathrm{~ms}$ ) obtained by the double-frame/ single-pulsed method is shown in Fig. 2.

The size of the field of view (FOV) was $172 \times 215 \mathrm{~mm}^{2}$ and the mean water level is $\mathrm{z}=0$ and the location of wave gage is $x=0$. The $32 \times 32$ pixels interrogation windows corresponded to

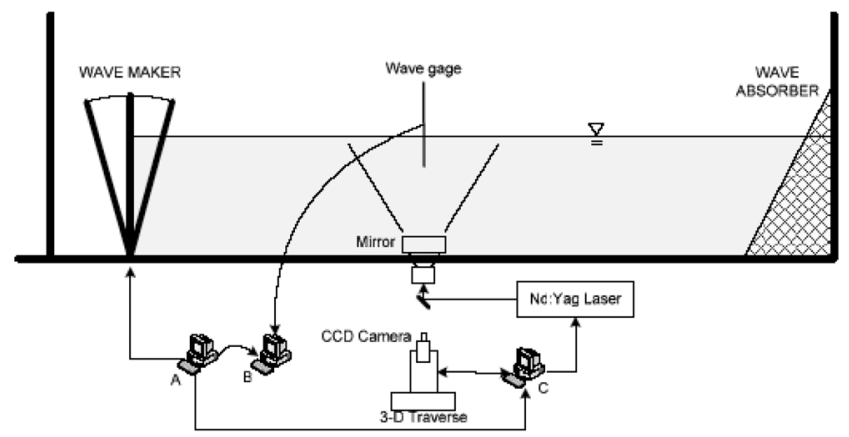

Fig. 1 Schematic sketch of the experimental set-up

Computer A: to control the wave maker and trigger the PIV system

Computer B: to take data from wave the gage

Computer C: to control the laser and CCD camera

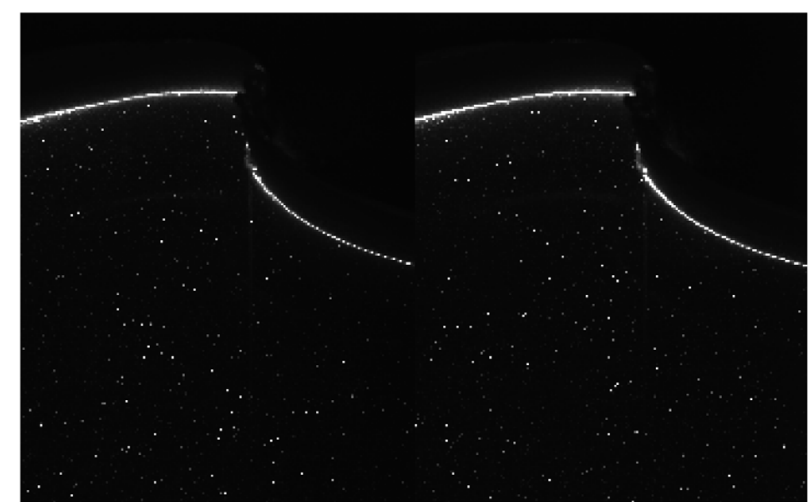

Fig. 2 Pair of images taken by the double-frame/single-pulsed method

a spatial resolution of $2.72 \times 2.72 \mathrm{~mm}^{2}$ and the time separation(dt) between the first and second laser pulses was $3.0 \mathrm{~ms}$.

The adaptive multi-pass algorithm was applied to reduce faulty vectors. Because this method has shifted an interrogation area to the location where particles moved, the stronger cross-correlation can be taken. Once the velocity vectors have been calculated in the interrogation area (32x32pixels) with $50 \%$ overlap, spurious false vectors were eliminated by the median filter (Westerweel, 1993). The left-over empty spaces were filled-up with interpolated vectors and smoothed by a simple $3 \times 3$ smoothing filter to reduce noise.

A series of experiments using the PIV system was conducted to measure the water particle velocities of irregular and rogue waves. The set-up is shown schematically in Fig. 1 , where $x$ is the horizontal coordinate positive in the direction of wave propagation with $x=0$ at the wave maker and $z$ is positive upward. Free surface elevations of irregular and rogue waves were measured at $620 \mathrm{~cm}$ and $800 \mathrm{~cm}$, respectively, from the wave maker, respectively. The irregular wave trains with JONSWAP spectrum were generated with four different significant wave heights. The water depth in the wave tank was maintained at $90 \mathrm{~cm}$.

Table 1 shows the experimental conditions for irregular and rogue wave test. The JONSWAP spectrum was used for generation of the waves of Cases PH1, PH2, and PH3. In order to generate different maximum height waves of irregular, the maximum crest part of the original input signal artificially adjusted according to the method of Zou and Kim (2000). If the maximum height is much greater than two times the significant wave height of the record and the ratio of crest height $H_{c}$ to significant wave height $H_{s}$ is greater than 1.25, it can be called a rogue wave or freak wave (Olagnon and Iseghem, 2001). The maximum height wave of 
Case PH3 was satisfied with these criteria of rogue waves. $H$, $T$, and $L$ in Table 1 are the wave height, period, and wave length of highest crest height wave in irregular wave train, respectively. The factor of asymmetric wave which is the ratio of the falling part (rear part) of crest to the rising part (front part) of crest is indicated as $F / R$.

Table 1 Experimental conditions for irregular waves

\begin{tabular}{ccccccc}
\hline \hline CASE & $H S(\mathrm{~cm})$ & TS $(\mathrm{s})$ & $H(\mathrm{~cm})$ & $H / H S$ & $H_{c} / H S$ & $F / R$ \\
\hline PH1 & 6.63 & 1.25 & 14.11 & 2.13 & 1.20 & 0.93 \\
PH2 & 6.995 & 1.27 & 15.11 & 2.16 & 1.22 & 1.24 \\
PH3 & 7.43 & 1.19 & 16.09 & 2.17 & 1.25 & 1.61 \\
\hline
\end{tabular}

\section{Experimental Procedure and Data Processing}

Irregular and rogue waves were generated in the 2-D wave tank. Before conducting a rogue wave experiment, PIV system accuracy was verified by comparing them to Stokes wave theory (Choi et al., 2010). It was found that the results obtained by PIV system had a good agreement with wave theory results.

A series of irregular wave trains have been obtained to generate the rogue wave in the 2-D wave tank and to measure the water particle velocities using the PIV technique. The rogue wave may occur in the process of geometrical focusing, dispersion enhancement (Kharif et al., 2001) and wave-current interactions (Peregrine, 1976; Smith, 1976; Lavrenov, 1998) in the real field. A combination of the geometrical focusing and dispersion enhancement mechanism can also be considered as the process of forming an extreme wave (Wu and Nepf, 2002). Many researchers have suggested and developed the nonlinear theory for rogue waves over the last decade. However, the rogue wave which is an event of majestic individual waves and highly nonlinear phenomena is not fully understood with the present modeling of surface waves (Haver, 2004).

Two important characteristics of the rogue wave are considered for generating the rogue wave in the 2-D wave tank instead of in the real ocean. The first characteristic was the definition of a rogue wave. If the maximum height is more than twice as large as the significant wave height, and the ratio of crest height $H_{c}$ to significant wave height $H_{s}$ is greater than 1.25 , it can be called a rogue wave (Olagnon and Iseghem, 2001). The second important characteristic of the rogue wave is that the highly nonlinear wave is a strongly asymmetric wave. In the ocean, strong asymmetric waves may be generated by the wind whose pressure is higher on the back than on the front of the crest. The highly nonlinear wave was simulated numerically by imposing such pressure on the surface of a wave of a particular period (Cokelet, 1977). Myrhaug and Kjeldsen (1986) analyzed the field data of the extreme seas where many ships were lost and described the extreme wave, as "a shallow and relatively long trough followed by a high and relatively short crest", which is similar to a strongly asymmetric wave. Bonmarin and Kjeldsen (2001) investigated the effects of the geometric and kinematic properties of plunging waves. They concluded that the asymmetry of a wave was an important parameter which contributes to the danger of breaking waves.

To generate the rogue wave in the 2-D wave tank, the irregular wave train is designed with JONSWAP spectrum and the distortion method was applied to the maximum wave of an irregular wave train (Funke and Mansard, 1982). Amplitude distortion was intended to increase the crest height and reduce the trough height but kept the amplitude spectrum and changed the phase spectrum only. The time distortion was utilized to make the duration of the trough longer and that of the crest shorter but its time period remained. Crest distortion was employed to move the location of the highest wave crest forward, and therefore, the front steepness would be increased. The largest wave of Case PH3 met the criteria for a rogue wave. Fig. 3 shows the whole time series and strong asymmetric rogue wave.

The total acceleration derivative is composed of the local acceleration and the convective acceleration terms. Totalacceleration can be obtained by the following equations;

$$
\frac{d u}{d t}=\frac{\partial u}{\partial t}+u \frac{\partial u}{\partial x}+w \frac{\partial u}{\partial z}
$$

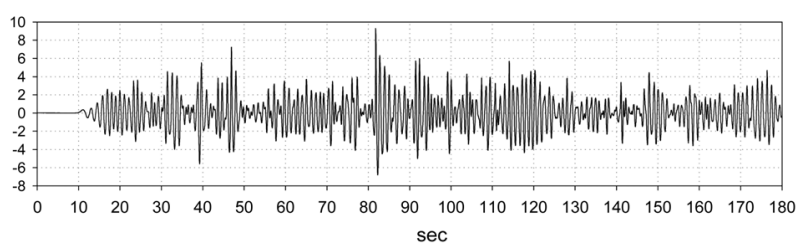

(a) Whole time series for Case PH3, total 155 number of waves

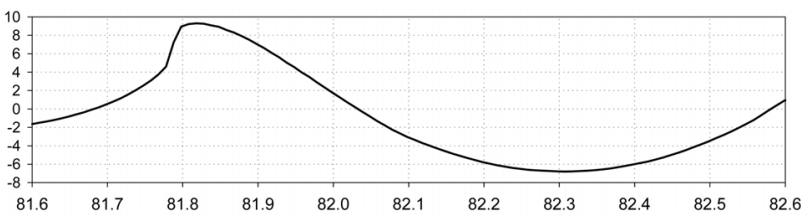

(b) Time series of the highest elevation wave for Case PH3

Fig. 3 Time series of irregular waves for Case PH3, $T_{s}=1.19 \mathrm{~s}$, $H_{s}=7.43 \mathrm{~cm}$, the highest elevation wave height, $H=16.09 \mathrm{~cm}$, $\mathrm{T}=0.9 \mathrm{~s}, \mathrm{H} / \mathrm{H}_{\mathrm{s}}=2.17, \mathrm{H}_{\mathrm{c}} / \mathrm{H}_{\mathrm{s}}=1.25$ 


$$
\frac{d w}{d t}=\frac{\partial w}{\partial t}+u \frac{\partial w}{\partial x}+w \frac{\partial w}{\partial z}
$$

where $d u / d t$ and $d w / d t$ represent total horizontal and vertical accelerations of a water particle, respectively.

The local acceleration fields were computed by applying the centered difference scheme to measured velocity fields. The equation of a center difference method for a local acceleration is represented as follows:

$$
a_{t}=\frac{\left(u_{t+1}-u_{t-1}\right)}{2 \cdot \Delta t}
$$

\section{Experimental Results for Rogue Wave Kinematics}

The three irregular wave trains are generated from the JONSWAP spectrum with significant wave heights of $6.6 \mathrm{~cm}$, $7 \mathrm{~cm}$, and $7.4 \mathrm{~cm}$, and the mean wave period $T_{m}$ of $1.2 \mathrm{~s}$ and the peak enhancement factor $r$ of 6.5 in the 2-D wave tank. The maximum wave was defined as the highest wave crest in the wave train. The maximum waves of Case PH3 was a rogue wave which satisfied with two criteria of a rogue wave. Twenty eight instantaneous velocity fields were obtained by measuring velocities of neighboring the maximum wave crest with the time step of $75 \mathrm{~ms}$. The three prediction methods of Wheeler stretching (Wheeler, 1970), linear extrapolation and modified stretching (Kim et al, 1997) were used to calculate kinematics of maximum wave in the irregular waves and were compared with experimental results. The solution of equivalent height with the third-order Stokes wave is also compared with the experimental results. The measured velocities are represented with the normalized value; i.e., the measured vertical position $Z$ was normalized by water depth $d$ and $u$ and $w$ were normalized by the phase velocity $v_{p}$ of the maximum wave. Fig. 4 shows the horizontal and vertical velocities under maximum wave crest for Case PH3. The maximum wave of Case PH3 has satisfied with criteria of rogue wave definition. Fig. 4(a) shows comparisons of measured horizontal velocities and four wave kinematics predictions under the wave crest of Case PH3. Note that Wheeler stretching prediction for the measured horizontal velocity in Case $\mathrm{PH} 3$ is underestimated and the prediction of linear extrapolation reasonably agreed well with measurements. In Fig. 4(a), the modified stretching method overpredicted the measured horizontal velocities below normalized vertical measuring position $Z / d=0.06$ for Case PH3. The equivalent height wave kinematics which is derived from the third-order Stokes wave theory is also compared with the measured horizontal velocities for Case PH3. The results of equivalent wave method for Case $\mathrm{PH} 3$ are overestimated the measurements below normalized vertical measuring position $Z / d=0.04$ and underestimated experimental data above normalized vertical measuring position $Z / d=0.04$. Fig. $4(\mathrm{~b})$ represents the comparisons of measured vertical velocities and three kinematics predictions under the wave crest of Case PH3. The Wheeler stretching was underestimated the vertical velocities, and the linear extrapolation and the modified stretching method agreed very well up to normalized vertical measuring position $\mathrm{Z} / \mathrm{d}=0.08$.

The local and convective accelerations of rogue wave kinematics (Case PH3) are represented in Fig. 5 and Fig. 6. The local and convective accelerations were computed using PIV results under the maximum wave crest of the three irregular wave trains. The centered difference method was applied for the local acceleration with $d t=75 \mathrm{~ms}$ and theconvective acceleration with $d x=d z=8 \mathrm{~mm}$. The horizontaland vertical components are investigated for both

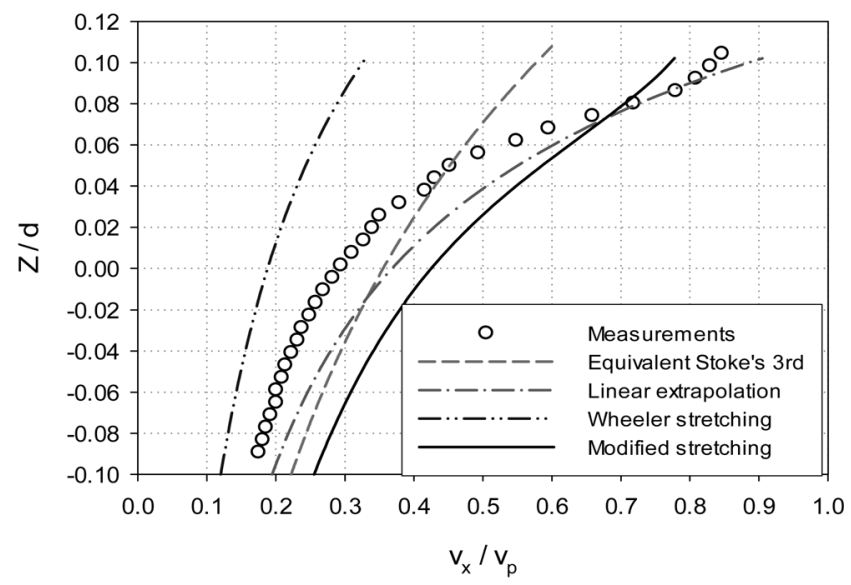

(a) Horizontal velocities under the rogue wave crest

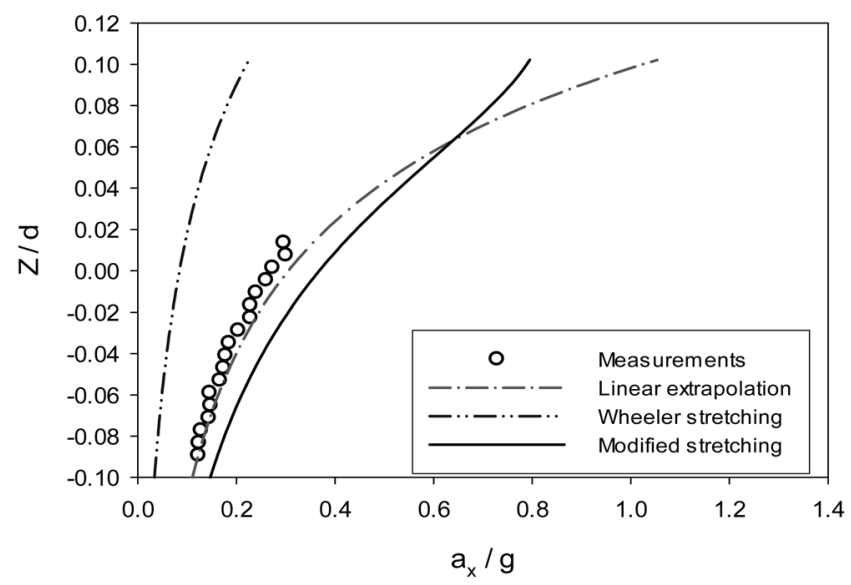

(b) Vertical velocities under the rogue wave crest

Fig. 4 Velocities under the rogue wave crest in Case PH3 
accelerations. The Wheeler stretching, the linear extrapolation, and the modified stretching, for calculating were used to compute the wave kinematics of irregular wave and compare with experiments. The local accelerations were presented in normalized values; i.e., measured vertical position $Z$ was normalized by water depth $d$ and local accelerations were normalized by gravity acceleration.

Figure 5(a) shows horizontal local accelerations from measurement-based computation and three wave kinematics predictions under the wave crest of Case PH3. The Wheeler stretching prediction was underestimated as shown in Fig. 5(a). Predictions using the linear extrapolation and the modified stretching showed a relatively good agreement with values of measurement-based computation for horizontal local acceleration. Vertical local accelerations from measurement-based computation and four kinematics predictions under the wave crest are shown in Fig. 5(b). The Wheeler stretching prediction underestimates vertical local accelerations as same as the horizontal acceleration. The modified stretching and the linear extrapolation showed a relatively good agreement with the vertical local accelerations from measurement-based computation. Note that the magnitudes of horizontal and vertical local accelerations obtained by PIV measurement were similar under the wave crest for Case $\mathrm{PH} 3$.

For horizontal convective accelerations of Case PH3, all predictions were agreed well with experimental results up to the mean water level. Linear extrapolation showed the opposite sign with experimental results near the wave crest. Modified stretching prediction shows good agreement with the results from measurement-based computation. Magnitude of horizontal convective acceleration for Case $\mathrm{PH} 3$ is negligibly small up to $Z / d=0.04$ but the magnitude is rapidly increased above $Z / d=0.04$. In Fig. $6(\mathrm{~b})$ for vertical convective accelerations, four predictions moderately agreed well with experimental results up to the mean water level. Linear extrapolation showed very good agreement with experimental results overall water depth.

\section{Conclusions}

In order to understand rogue wave kinematics, a series of experiments was conducted in the 2-D wave tank. The velocity fields obtained by PIV were used to compute the water particle accelerations using the central difference scheme. The local term and convective term of total acceleration were computed from PIV measurements. Thelaboratory rogue wave was generated in the 2-D wavetank and measured velocities near the wave crest zone

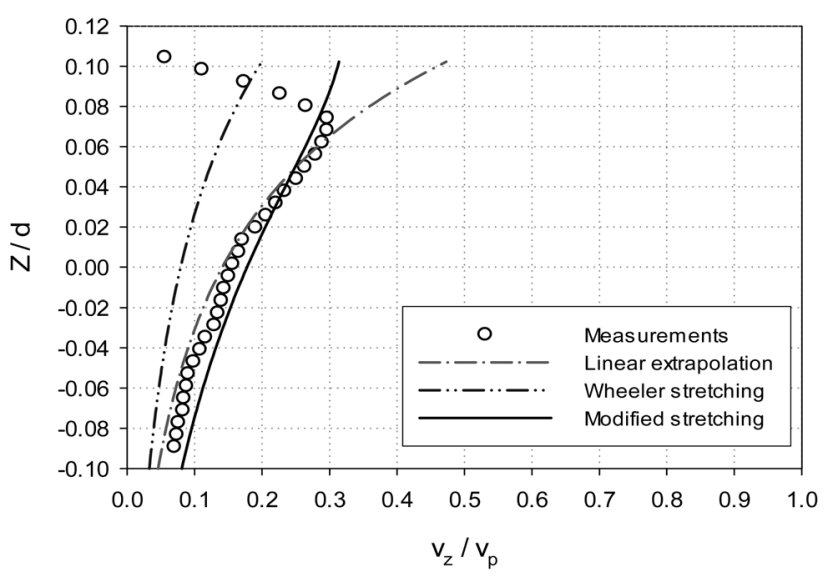

(a) Horizontal local accelerations under the rogue wave crest

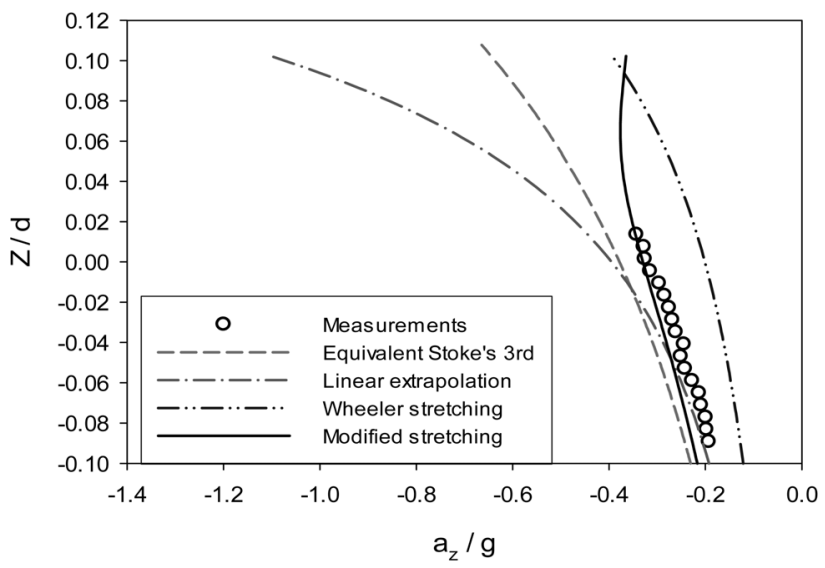

(b) Vertical local accelerations under the rogue wave crest

Fig. 5 Local accelerations under the rogue wave crest for Case PH3

using PIV system. The measured velocities were used to compute the rogue wave accelerations with the central difference scheme.

The rogue wave was obtained from three different irregular wave trains. The wave particle velocities were measured at the maximum wave height of irregular wave trains which satisfied the rogue wave category. The velocity and acceleration under the wave crest were focused. From the comparison of the experimental results and predictions for rogue waves, the following conclusions may be drawn.

(1) The rogue wave could be generated in the 2-D wave tank.

(2) The Wheeler stretching method underestimated the wave kinematics of the irregular waves. The modified stretching method could predict the maximum values of rogue wave kinematics well.

(3) The magnitude of vertical convective acceleration under 


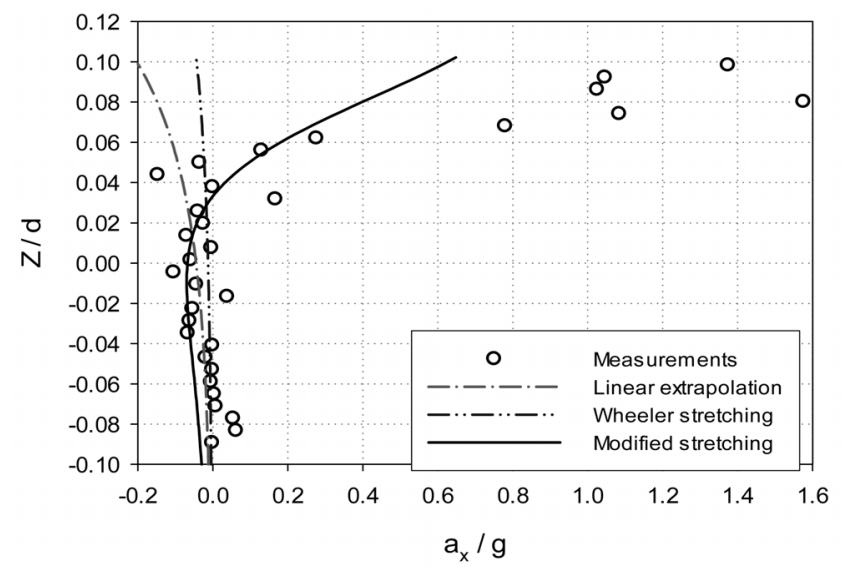

(a) Horizontal convective accelerations under the rogue wave crest

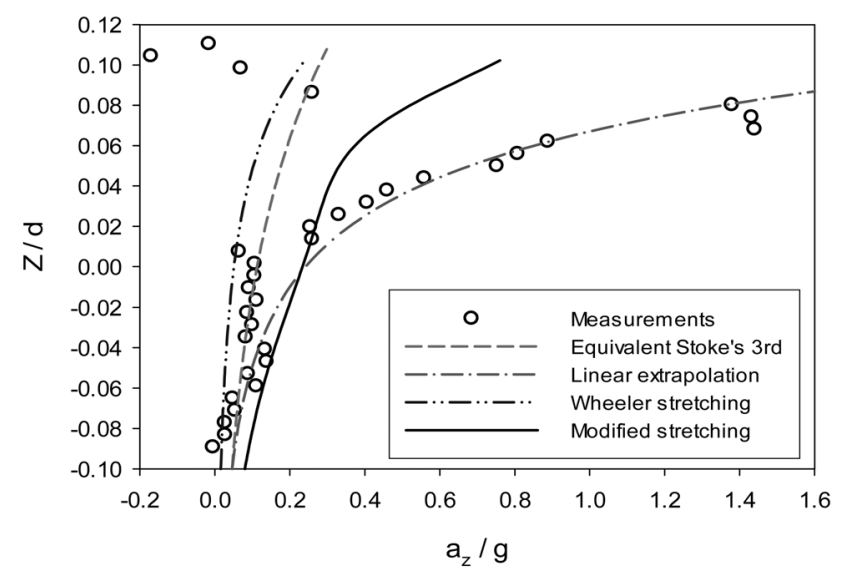

(b) Vertical convective accelerations under the rogue wave crest

Fig. 6 Convective accelerations under rogue wave crest for Case $\mathrm{PH} 3$

the rogue wave crest is similar to the horizontal convective acceleration.

(4) In the experimental results of rogue wave kinematics, the magnitude of convective acceleration under the wave crest is rapidly increased near the wave crest.

(5) The convective acceleration for the rogue wave has the similar magnitude with local acceleration.

(6) The total acceleration of water particle under the rogue wave crest can be estimated approximately to be twice of gravity acceleration.

\section{Acknowledgement}

This research was supported by Basic Science Research Program through the National Research Foundation of Korea(NRF) funded by the Ministry of Education, Science and Technology (KRF-2008-331-D00725)

\section{References}

Airy, G.B. (1845). “Tides and waves," Encyclopedia Metropolitana, London, 5, pp 241-396.

Bonmarin, P. and Kjeldsen, P. (2001). "Some Geometric and Kinematic Properties of Breaking Waves," In: Olagnon, M., and Athanassoulis, G. (Eds.) Rogue Waves 2000. Ifremer, Brest, pp 169-180.

Choi H.J., Jung K.H., Suh S., Jo H.J. and Choi H.S. (2010). "Experimental Study on Nonlinearity Characteristics Near the Free Surface in the Regular Wave Condition" Journal of Ocean Engineering and Technology, Vol 24, No 1, pp 1-9

Clamond, D. and Grue, J. (2002). "Interaction between Envelop Solitons as a Model for Freak Wave Formulations-part 1: Long Time Interaction," Comptes Rendus. Mecanique, 330, pp 575-580.

Clauss, G.F. (2002). "Dramas of the Sea: Episodic Waves and Their Impacts on Offshore Structures," Applied Ocean Research, 24, 147-161.

Cokelet, E.D. (1977). "Steep Gravity Waves in Water of Arbitrary Uniform Depth," Philosophical Transactions of the Royal Society of London Series A-Mathematical Physical and Engineering Sciences, 286 (1335), pp 183-230.

Dean, RG (1990). "Freak Wave: A Possible Explanation," In: Tørum, A., and Gudmestad, O.T. (Eds.) Water Wave Kinematics, NATO ASI Series, Kluwer, Dordrecht, The Netherlands, pp 609-612.

Draper, D. (1965). "Freak Ocean Waves," Marine Observer, 35, pp 193-195.

Dysthe, K.B. and Trulsen, K. (1999). "Note on Breather Type Solutions of the NSL as a Model for Freak-Waves," Physica Scripta, T82, pp 48-52.

Faulkner, D. (2001). "Rogue Waves - Defining Their Dharacteristics for Marine Design," Rogue Waves 2000, Ifremer, Brest, pp 3-18.

Funke, E.R. and Mansard, E.P.D. (1982). "The Control of Wave Asymmetries in Random Waves," Proc 18th International Conference on Coastal Engineering, Cape Town, South Africa, pp 725-744.

Grue, J. (2002). “On Four Highly Nonlinear Phenomena in Wave Theory and Marine Hydrodynamics," Applied Ocean Research, 24, pp 261-274.

Haver, S. (2001). "Evidences of the Existence of Freak Waves," Rogue Waves 2000. Ifremer, Brest, pp 129-140.

Henderson, K.L., Peregrine, D.H. and Dold, J.W. (1999). "Unsteady Water Wave Modulations: Fully Nonlinear Solutions and Comparison with the Nonlinear 
Schrödinger Equation," Wave Motion, 29, pp 341-361.

Kharif, C., Pelinovsky, E., Talipova, T. and Slunyaev, A. (2001). "Focusing of Nonlinear wave Groups in Deep Water," JETP Letters, 73 (4), pp 170-175.

Kim, N.S. and Kim, C.H. (2003). "Simulation of Draupner Freak Wave Impact Force on a Vertical Truncated Cylinder," Int J of Offshore and Polar Engineering, 13 (4), pp 260-265.

Kim, C.H., Xu, Y. and Zou, J. (1997). "Impact and Nonimpact on Vertical Truncated Cylinder due to Strong and Weak Asymmetric Wave," Int J of Offshore and Polar Engineering, 7 (3), pp 161-167.

Kjeldsen, P. (2001). "A Sudden Disaster-in Extreme Waves," Rogue Waves 2000. Ifremer, Brest, pp 19-35.

Jansen, P.A.E.M. (2003). "Nonlinear Four-Wave Interactions and Freak Waves," J of Physical Oceanography, 33, pp 863-884.

Lavrenov, I.V. (1998). "The Wave Energy Concentration at the Agulhas current off South Africa," J of Natural Hazards, 17, pp 117-127.

Lemire, J. "Freak Wave Rocks Cruise," http://www.nydailynews.com/front/story/300826p-257523 c.html, Accessed April. (2005).

Mastroianni, M. "Giant Wave Hits Semester at Sea Ship," http://www.pittnews.com/vnews/display.v/ART/2005/0 1/28/41f9e1e0b91d4, Accessed March. (2005).

Met Office (1996). "Hurricane 'Luis', the Queen Elizabeth 2, and a Rogue Wave," Marine Observer, 66 (333), pp 134-137.

Mori, N. and Yasuda, T. (2001). "Effects of High-Order Nonlinear Wave-Wave Interactions on Gravity Waves," Rogue Waves 2000. Ifremer, Brest, pp 229-244.

Myrhaug, D. and Kjeldsen, S.P. (1986). "Steepness and Asymmetry of Extreme Waves and the Highest Waves in Deep Water," Ocean Engineering, 13 (6), pp 549-568.

Olagnon, M. and Van Iseghem, S. (2001). "Some Cases of Observed Rogue Waves and an Attempt to Characterize Their Occurrence Conditions," Rogue Waves 2000. Ifremer, Brest, pp 105-116.

Onorato, M., Osborne, A.R. and Serio, M. (2002). "Extreme Wave Events in Directional, Random Oceanic Sea State," J of Physics of Fluids, 14 (4), L25-L28.
Osborne, A.R., Onorato, M. and Serio, M. (2000). "The Nonlinear Dynamics of Rogue Waves and Holes in Deep-Water Gravity Wave Trains," J of Physics Letters A, 274, pp 386-393.

Peregrine, D.H. (1976). "Interaction of Water Waves and Currents," Advances in Applied Mechanics, 16, pp 9-117.

Rosenthal, W. and Lehner, S (2004). "Results from the MAXWAVE project," Proc the 23th OMAE conference, Vancouver, Canada.

Smith, R. (1976). "Giant Waves," J of Fluid Mechanics, 77, pp 417-431.

Smith, S.F. and Swan, S. (2002). "Extreme Two-Dimensional Water Waves: An Assessment of Potential Design Solutions," Ocean Engineering, 29 (4), pp 387-416.

Trulsen, K. and Dysthe, K.B. (1997). "Freak Waves-A Three Dimensional Wave Simulation," Proc of the 21th Symposium on Naval Hydro-dynamics, The National Academy of Sciences, Washington, D.C., pp 550-560.

Tulin, M.P. and Waseda, T. (1999). "Laboratory Observations of Wave Group Evolution, Including Breaking Effects," J of Fluid Mechanics, 378, pp 197-232.

Westerweel, J. (1993). Digital Particle Image Velocimetry - Theory and Application, Ph.D Dissertation, Delft University, The Netherlands.

Wheeler, J.D. (1970). "Method for Calculating Forces Produced by Irregular Waves," J of Petroleum Technology, 249, pp 359-367.

$\mathrm{Wu}$, C.H. and Nepf, H. (2002). "Breaking Criteria and Energy Losses for Three-Dimensional Wave Bbreaking," Geophysical Research, 107 (c10), (41) pp 1-18.

Wu, C.H. and Yao, A. (2004). "Laboratory Measurements of Limiting Freak Waves on Currents," Geophysical Research, 109(c12002), pp 1-18.

Zou, J. and Kim, C.H. (2000). "Generation of Strongly Asymmetric Wave in Random Seaway," Proc of the 10th International Offshore and Polar Engineering Conference, Seattle, pp 95-100.

2011년 6월 20일 원고 접수 2011년 6월 27일 심사 완료 2011년 6월 28일 게재 확정 